\title{
Comparison of algorithms for non-linear inverse 3D electrical tomography reconstruction
}

\author{
Marc Molinari ${ }^{1}$, Simon J Cox ${ }^{1}$, Barry $\mathbf{H}$ Blott $^{2}$ and Geoffrey J Daniell ${ }^{2}$ \\ ${ }^{1}$ Department of Electronics and Computer Science, University of Southampton, Southampton, \\ SO17 1BJ, UK \\ ${ }^{2}$ Department of Physics and Astronomy, University of Southampton, Southampton, \\ SO17 1BJ, UK \\ E-mail: sc@ecs.soton.ac.uk
}

Received 13 July 2001, in final form 10 August 2001

Published 28 January 2002

Online at stacks.iop.org/PM/23/95

\begin{abstract}
Non-linear electrical impedance tomography reconstruction algorithms usually employ the Newton-Raphson iteration scheme to image the conductivity distribution inside the body. For complex 3D problems, the application of this method is not feasible any more due to the large matrices involved and their high storage requirements. In this paper we demonstrate the suitability of an alternative conjugate gradient reconstruction algorithm for 3D tomographic imaging incorporating adaptive mesh refinement and requiring less storage space than the Newton-Raphson scheme. We compare the reconstruction efficiency of both algorithms for a simple 3D head model. The results show that an increase in speed of about $30 \%$ is achievable with the conjugate gradientbased method without loss of accuracy.
\end{abstract}

Keywords: efficient non-linear 3D electrical impedance tomography reconstruction, three-dimensional adaptive mesh refinement, conjugate gradient solver

\section{Introduction}

The generation of electrical conductivity tomograms from external measurements is approaching the limits of development using 2D models (Holder and Brown 1993, Blott et al 1998, Vauhkonen et al 2001). The principals of the reconstruction method are the incorporation of a statistical test of acceptance for how well the reconstructed image accounts for the data bearing in mind the noise in the measurements and the creation of a measure of the image characteristics. The latter 'smoothness' measure is essential to ensure that only the features in the image that can be relied upon are retained. The inverse problem is then 
solved with a non-linear Newton-Raphson (NR) algorithm. The calculation of the tomograms is implemented with the aid of a finite element mesh in which, to keep computing time down, the element size is adapted to match the scale of image variations (Molinari et al 2002).

In three-dimensional medical electrical impedance tomography the complexity of body shapes and components requires a large number of mesh elements which raises new computer capacity problems on single processor systems. Either the mesh discretization becomes too coarse to obtain images unaffected by the element size or it causes the computer to run out of memory with the commonly used algorithms. There exist two ways of addressing this capacity problem: (1) to parallelize and thus distribute the problem onto several processors (Blott et al 2000) and (2) to investigate alternatives to Newton-Raphson for the optimization problem-we will focus on a non-linear conjugate gradient (CG) approach.

After reviewing, in the next section, the requirements for efficient reconstruction algorithms on electrical impedance tomography (EIT), we take the second approach and give details about our implementation of a Newton-Raphson-based algorithm as well as a new conjugate-gradient algorithm to solve the $3 \mathrm{D}$ inverse problem. In section 5 , we compare the efficiency of these two algorithms applied to the reconstruction of a simple head model. Finally, we draw our conclusions in section 6.

\section{Review of algorithmic requirements for medical imaging}

Table 1 summarizes the major requirements for algorithms applicable to medical EIT. The model of an object created in the pre-processing stage affects the flexibility of the algorithm which impacts on the whole reconstruction process. Accuracy and speed are mainly affected by numerical code implementations. After a satisfactory solution is obtained, its visual representation in a medically useful format should not be overlooked. Visualization characteristics in the post-processing stage often depend on the initial model and the reconstruction algorithm used.

Table 1. Requirements for an efficient EIT reconstruction algorithm for medical applications (extended from Molinari et al 2001b).

\begin{tabular}{ll}
\hline Flexibility & Accurate modelling of complex 2D and 3D geometries \\
& Easy application to differing electrode types \\
& Possibility of node relocation for dynamic imaging \\
Accuracy & Usage of high-quality domain discretization \\
& Robustness with respect to noise \\
& Minimal influence of algorithmic constraints on solution accuracy \\
& Suitable algorithm for the problem's non-linear nature \\
Speed & Application of sparse matrix storage schemes and solver techniques \\
& $\begin{array}{l}\text { Algorithm with problem-adaptive mesh density } \\
\text { Parallelization of code }\end{array}$ \\
& Extraction of medically significant features from image \\
& Display of slices, surfaces, volumes
\end{tabular}

Some of the requirements listed in table 1 have been addressed in the context of 3D EIT in previous studies, others still remain to be investigated. Amongst these are:

- accurate boundary shape representation as investigated for $2 \mathrm{D}$ problems by Jain et al (1997), 
- modelling of general object shapes, such as the head (Gibson et al 2000),

- high reconstruction times caused by large dense matrices,

- resolution inaccuracies caused by deficient element quality (Vauhkonen et al 1999),

- accounting for the influence of off-plane structures in 3D reconstructions (Metherall et al 1996).

\section{Reconstruction algorithm}

Our reconstruction of a conductivity distribution $\sigma$ within a volume conductor $\Omega$ by means of EIT is based on the minimization of a functional $\Phi$, which employs the statistical $\chi^{2}$ criterion for goodness of fit. It works on the difference between computed and measured electrode voltages, $U_{i}^{\text {comp }}-U_{i}^{\text {meas }}$ for all measurements $i=1 \ldots M$ and accounts for the measurement error $\delta U_{i}^{\text {meas }}$. A well-defined reconstruction is ensured by the image smoothness term $|\nabla \log \sigma|^{2}$ :

$$
\begin{gathered}
\Phi=\sum_{i=1}^{M}\left(\frac{U_{i}^{\text {comp }}-U_{i}^{\text {meas }}}{\delta U_{i}^{\text {meas }}}\right)^{2}+\lambda \int_{\Omega}|\nabla \log \sigma|^{2} \mathrm{~d}^{3} x \\
=\chi^{2}+\lambda \Psi .
\end{gathered}
$$

This system is equivalent for two- and three-dimensional problems. A more detailed discussion of this functional and the determination of the parameter $\lambda$ can be found in Blott et al (1998) and Molinari et al (in this issue).

Since there exist no analytical solutions to the generalized problem, we employ the wellstudied finite element method (Burnett 1987) to discretize $\Omega$ for a numerical solution. We use tetrahedral elements with piecewise linear base functions for the potentials and a constant conductivity parameter. This choice reduces the size of the matrices involved and therefore ensures an efficient reconstruction. The main advantages of the finite element discretization are its flexibility in terms of geometry and application of different types of boundary conditions imposed by the electrodes used. These advantages outbalance by far the disadvantage of the rather complex coding of finite element programmes.

To minimize (1) with respect to the conductivity distribution $\sigma$, we initially employ the non-linear Newton-Raphson iteration scheme for which Yorkey et al (1987) showed fastest convergence out of a number of 2D EIT reconstruction algorithms.

This iterative algorithm updates an initial homogenous conductivity guess by $\Delta \sigma$ during each iteration according to the following formula:

$$
\left(J^{T} J+\lambda R\right) \Delta \sigma=J^{T}\left(\frac{U^{\text {meas }}-U^{\text {comp }}}{\delta U^{\text {meas }}}\right)+\lambda r .
$$

The solution of this system involves the construction and Cholesky decomposition of the large dense sensitivity matrix $J^{T} J+\lambda R$ of size $n^{2}$ where $n$ is the number of finite elements. $J$ denotes the Jacobian weighted by the measurement uncertainties $\delta U^{\text {meas }} ; R$ and $r$ are a sparse matrix and a vector respectively, both introduced by the image smoothness term in (1). While $R$ is constant for a mesh and has to be computed only once, $J$ and $r$ require updating during each iteration step.

The repeated computation and subsequent Cholesky decomposition of the dense sensitivity matrix makes the Newton-Raphson approach unsuitable for large complex 3D problems as $n$ can easily exceed 5000, which corresponds to a matrix size of $25 \times 10^{6}$ or a memory requirement of $200 \mathrm{MB}$ to store the matrix alone.

An alternative approach is the application of a non-linear conjugate gradient (CG) algorithm. This type of algorithm is well studied (Hake 1992) and recognized as one of 


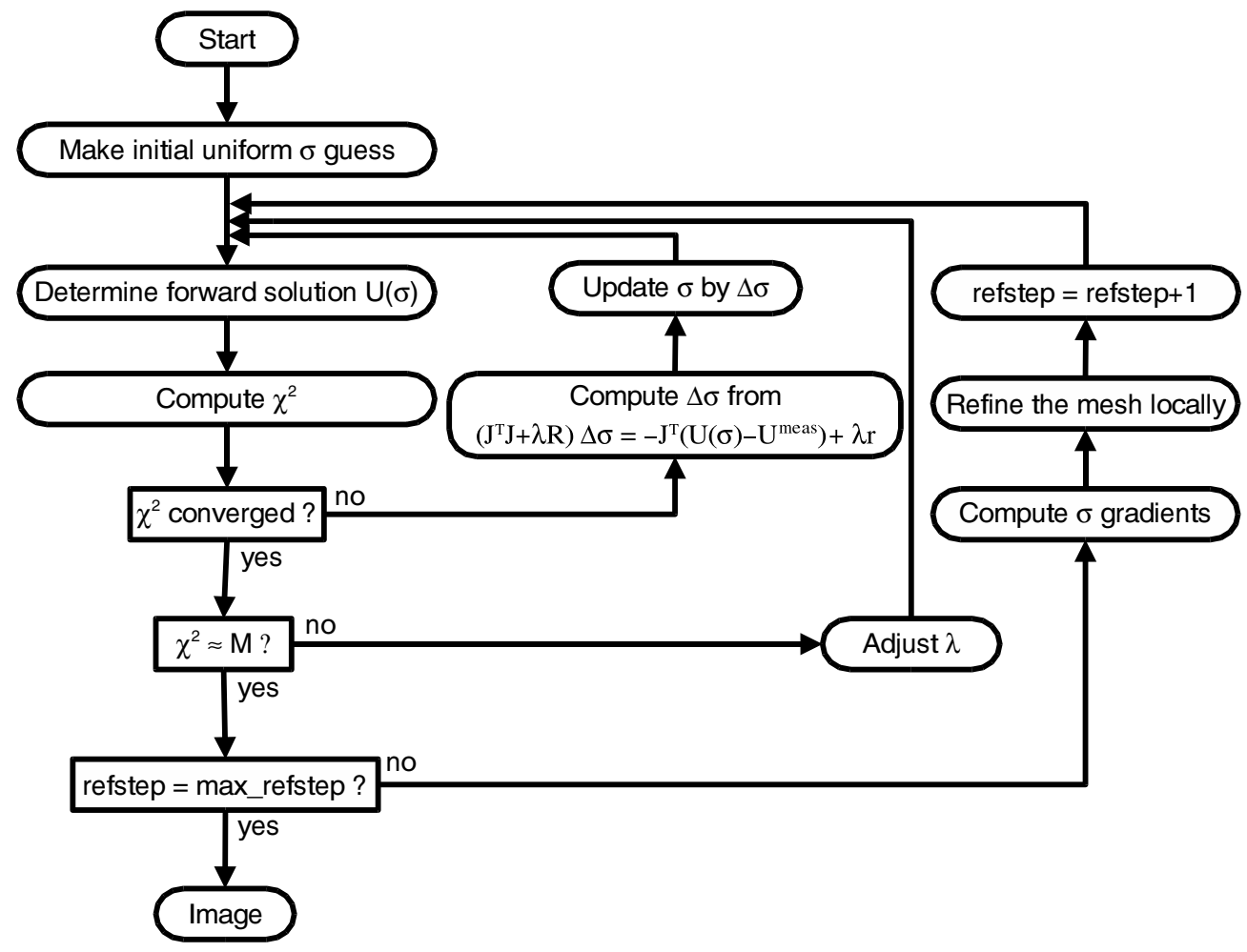

Figure 1. Outline of Newton-Raphson imaging algorithm with adaptive mesh refinement in the reconstruction process.

the most suitable numerical algorithms for complex ill-conditioned large-scale applications (Golub and O'Leary 1989, Arridge and Schweiger 1998). The CG method searches for a minimum of the functional $\Phi$ by taking conjugate search directions for every iteration step and requires the computation of the gradient of $\Phi$ only, instead of the full second derivative. Different choices for finding the minimum along a search direction exist. The application of an inexact line search algorithm does avoid the necessity of computing the Jacobian for every iteration step. We implemented the CG algorithm with the Polak-Ribière parameter updating technique (see, e.g. Shewchuk 1994).

Figures 1 and 2 show the two algorithms with the auto-adaptive mesh refinement wrapper. Both algorithms terminate when the statistical criterion $\chi^{2} \approx M$ is satisfied, where $M$ is the number of independent measurements made and the targeted number of refinements is reached.

\section{Adaptive mesh refinement in $3 D$}

The speed and accuracy of the imaging process - the reconstruction of conductivities given only surface potential measurements-depend not only on the algorithm used, but also strongly on the underlying finite element discretization. We have shown that adaptive mesh refinement techniques can have a major impact on the efficient solution of the forward problem (Molinari et al 2001a) as well as on achieving the image resolution intrinsic in the data (Molinari et al in this issue). 


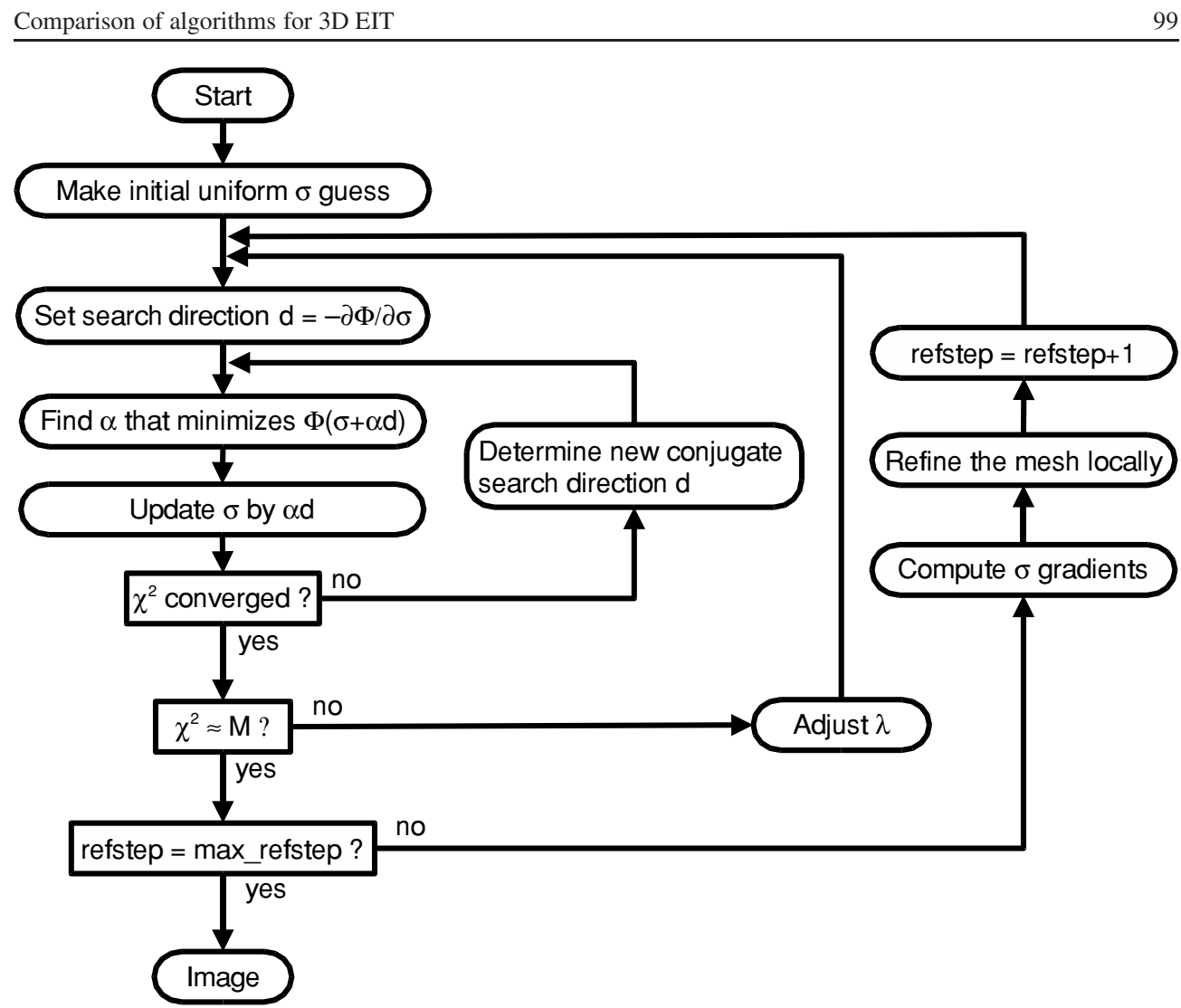

Figure 2. Outline of the non-linear conjugate gradient algorithm. Not shown are parameters such as termination criteria, Polak-Ribière updating and restarting criteria.

We have modified the NR and CG algorithms to include mesh refinement techniques. h-refinement - the subdivision of elements in the initial rather coarse mesh-is fast and offers a number of additional advantages, such as maintaining the underlying element geometry and focusing on selected areas (Burnett 1987), as well as being applicable to both the forward solution and the inverse problem. An error estimator in the former case and a conductivity gradient indicator in the latter case determine the elements to be refined. In three dimensions, the number of possible subdivisions of these selected elements is larger than in $2 \mathrm{D}$ thus allowing for more flexible control over local element density in the mesh as indicated in figure 3 .

Refinement for the inverse problem is carried out when the $\chi^{2}$ criterion is met after a number of iterations. Thus, the adaptive refinement code wraps around the actual algorithm. The flow diagram assumes that the initial mesh is not so coarse that $\chi^{2} \approx M$ cannot be reached. Tests at $80 \mathrm{~dB}$ signal-to-noise level have shown that after three refinement stages, no significant improvement is achieved by further refining the mesh.

To maintain the node connectivity within the mesh after adding a number of nodes, a local Delaunay re-triangulation (Golias and Dutton 1997) of the nodes within and adjacent to the refined elements is required. This can be carried out quickly and efficiently with, for example, the Qhull algorithm (Barber et al 1996).

Element-centred point insertion maintains the element boundaries and adds only three further elements within the existing one. This leads to the addition of relatively few elements at the expense of distorted element geometries. It is advisable to maintain element geometries 


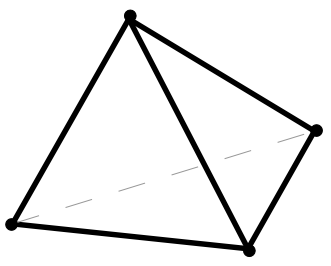

a)

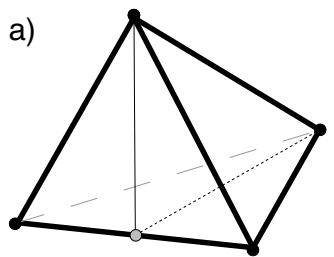

C)

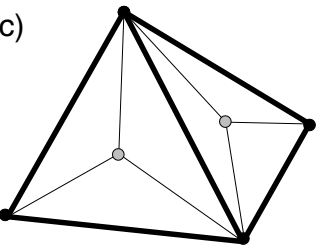

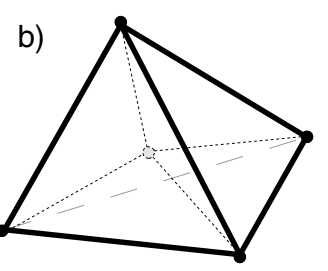

d)

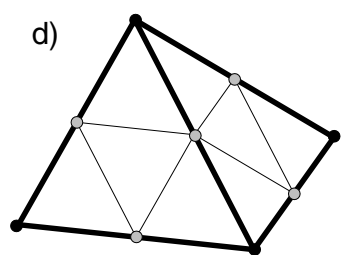

Figure 3. $h$-refinement by point insertion for 3D tetrahedra: (a) longest-edge bisection; (b) element-centred, (c) face-centred and (d) edge-centred point insertion.

which are as close as possible to equilateral tetrahedra. This so-called high-quality mesh avoids geometrical anisotropies causing numerical errors, especially in the region around the electrodes. The addition of six nodes on the edges of a selected element leads to the addition of seven elements of similar quality to the base element and should hence be preferred to the former two methods.

Mesh-conforming refinement is essential as the potential values of the forward solution computed at the nodes are used in the computation of the sensitivity matrix, $J$, in the NewtonRaphson procedure. This means that some of the nodes used in the computation of the forward solution must correspond to the nodes at which potential values are evaluated for the inverse solution. This is either done by using a base mesh and refining it according to its task (accurate forward solution or high conductivity boundary resolution) or combining these two meshes into one which incorporates both features. The latter possibility has the disadvantage of producing a large number of elements in places not specifically required for the inverse problem and hence causes longer computation times than necessary. Its advantages are the easier algorithmic implementation and the potential of quality improvement by relocating nodes in the centres of their adjacent neighbours, which is also known as Laplace smoothing.

\section{Simulation results}

We show results from reconstructions obtained with the algorithms described above on synthetic data. We use a simple dummy head model made up from a combination of solidconstructive-geometry objects, such as cylinders and spheres, and meshed with Netgen ${ }^{3}$. We simulated three conductivity parameters within the head mesh consisting of 40216 elements: the head itself filled with grey matter of $0.15 \mathrm{~S} \mathrm{~m}^{-1}$ (Foster and Schwan 1989) and a mouth region of an estimated $0.01 \mathrm{~S} \mathrm{~m}^{-1}$ as well as a blood clot of $0.67 \mathrm{~S} \mathrm{~m}^{-1}$ (Geddes and Baker 1967 ) in a central region behind the left eye of the patient. Figure 4 shows the simulated materials in front, side and top view and figure 5 shows slices at the indicated heights.

\footnotetext{
3 http://www.sfb013.uni-linz.ac.at/ joachim/netgen/.
} 

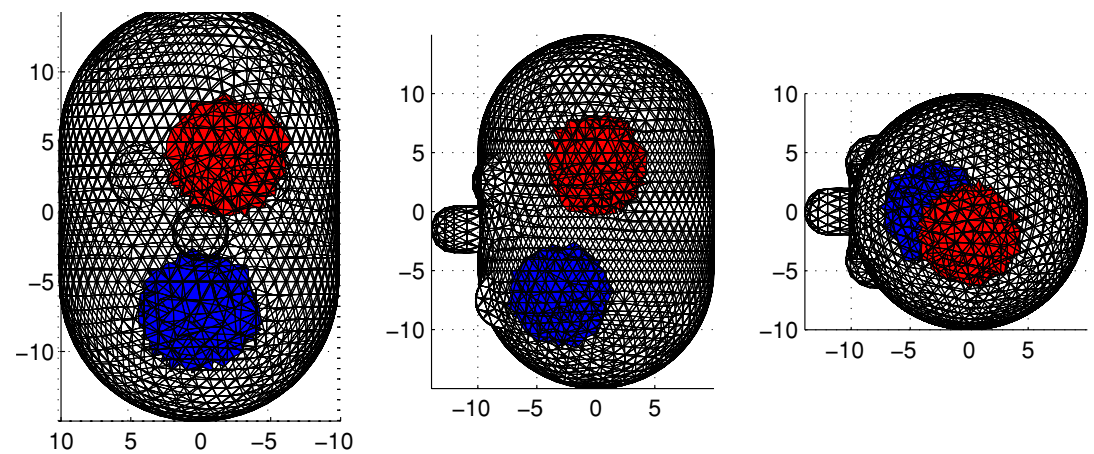

Figure 4. Front, left and top view of head dummy simulation with background conductivity (grey matter, $0.15 \mathrm{~S} \mathrm{~m}^{-1}$ ), mouth cave region (air, $0.01 \mathrm{~S} \mathrm{~m}^{-1}$ ) and object behind left eye (blood $\left.0.67 \mathrm{~S} \mathrm{~m}^{-1}\right)$.

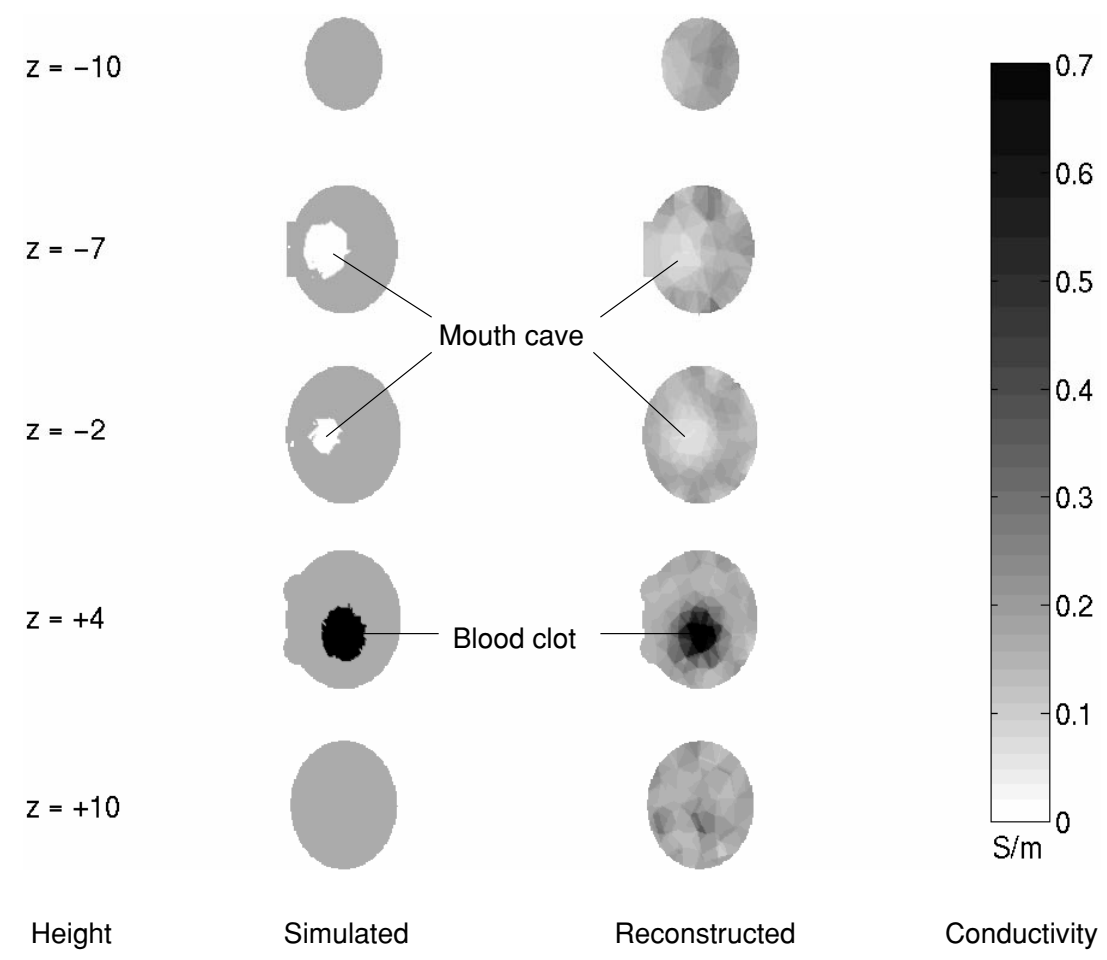

Figure 5. Slices through the head at the indicated heights showing the conjugate-gradient-based reconstructed conductivity distribution. The centre-left column represents the original values and the centre-right column displays the reconstructed values. Mouth cave and blood clot are clearly detectable. The range of reconstructed conductivites lies within the simulated values as indicated by the colour distribution. The Newton-Raphson result is visually indistinguishable.

To avoid shape mismatch between the electrodes applied to the surface in the forward case and those in the inverse case, we decided to employ point electrodes. The algorithm, however, is equally applicable to the complete electrode model as introduced by Somersalo et al (1992). Twenty-four electrodes in total have been applied — six on top, six at back and six on each side 
of the head-to ensure an appropriate coverage of the surface. Current injection is performed through both opposed and adjacent electrode pairs so that a total of 24 current patterns was used. This corresponds to a total of $(24 \times 21) / 2=252$ independent potential measurements. We assumed a signal-to-noise ratio of about $80 \mathrm{~dB}$ in the measurements, which is about the best achievable with current technology.

The initial mesh for the reconstruction consists of 5027 elements which corresponds to a spatial resolution of about $5.8 \%$. This model does not take into account the possible shunting effect by the skin and insulation by the skull barrier. These might affect the reconstruction from real measurements significantly. However, we are mainly concerned here with the performance aspects of the algorithm rather than the fidelity of the simulation.

\subsection{Imaging results}

Figure 5 shows the result of the $\mathrm{CG}$ algorithm which is visually indistinguishable from the NR algorithm. The quantitative analysis of the pixel error $E$ in table 2 reveals that both algorithms also perform equally well in terms of absolute conductivity reconstruction. $E$ is the averaged Frobenius norm of the difference between simulated and reconstructed conductivities sampled on a $100 \times 100 \times 100$ point grid.

Table 2. Comparison of Newton-Raphson (NR) and conjugate gradient (CG) algorithms.

\begin{tabular}{lll}
\hline & Newton-Raphson & Conjugate gradient \\
\hline Storage requirement $(\mathrm{MB})$ & 220 & 7.8 \\
Number of iterations & 9 & 172 \\
Solution time (min) & 16.9 & 12.2 \\
Average pixel error $E\left(\mathrm{~S} \mathrm{~m}^{-1}\right)$ & 0.0136 & 0.0143 \\
\hline
\end{tabular}

Although CG requires much less memory than Newton-Raphson, many more iterations have to be carried out to reach the statistical $\chi^{2}$ stopping criterion. The large number of iterations required increases the solution time and partly balances out the speed advantages. Nevertheless, our new CG-based approach achieved a $28 \%$ decrease in computing time. The calculation was performed using Matlab Release 12 on a $900 \mathrm{MHz}$ AMD Athlon processor PC with $1024 \mathrm{MB}$ of memory and running SuSE Linux 7.1 as operating system.

\section{Discussion and conclusions}

The iterative Newton-Raphson method is the method of choice for small-scale EIT problems. It exhibits robustness and high accuracy in the absolute imaging of synthetic data. When it comes to 3D imaging with arbitrarily shaped complex geometries, such as the head, this method becomes more and more unsuitable due to the large matrices involved. Storage and computing time rises as a factor of the number of elements involved and hence an alternative algorithm has to be found.

We have investigated the possible use of a non-linear conjugate-gradient-based algorithm. The results presented show that it is superior to Newton-Raphson in terms of speed and storage requirements as only the first derivative of the objective function needs to be computed. This leads to an increase in speed of about $28 \%$ for our example which consists of about 5000 elements only. As conjugate gradient time complexity scales with $O\left(n^{4 / 3}\right)$ (Shewchuk 1994), where $n=$ number of elements, compared to Newton-Raphson which scales with $O\left(n^{3}\right)$, significantly larger differences in reconstruction speed can be expected if even more elements are used. The fact that the conjugate gradient algorithm is easier to parallelize onto several 
processors (Takeda et al 2000) makes it a suitable candidate for 3D EIT reconstructions. It is in particular more suitable for complex large-scale problems when the number of elements increases above 10000 and the matrices involved require too much memory in the NewtonRaphson method.

To obtain fast reconstructions, not only the algorithm employed, but also technqiues such as adaptive mesh refinement play a crucial role. We have presented a number of possible refinement strategies for 3D tetrahedral finite elements which help to increase mesh density where required for forward and inverse problem solution. Further work is required to find the best way of obtaining the most suitable high-quality finite element mesh for image reconstruction and to investigate the usefulness of special numerical tools such as the Woodbury formula. These and the parallelization in 3D of the new conjugate gradient-based code will be the next problems to address in non-linear three-dimensional electrical impedance tomography.

\section{Acknowledgment}

MM is grateful to EPSRC for financial support.

\section{References}

Arridge S and Schweiger M 1998 A gradient-based optimisation scheme for optical tomography Optics Express 2 213-26

Barber C B, Dobkin D P and Huhdanpaa H 1996 The Quickhull algorithm for convex hulls ACM Trans. Math. Software 22 469-483

Blott B H, Cox S J, Daniell G J, Caton M J and Nicole D A 2000 High fidelity imaging and high performance computing in nonlinear EIT Physiol. Meas. 21 7-14

Blott B H, Daniell G J and Meeson S 1998 Nonlinear reconstruction constrained by image properties in electrical impedance tomography Phys. Med. Biol. 43 1215-24

Burnett D S 1987 Finite Element Analysis, from concepts to applications (Reading, MA: Addison-Wesley)

Foster K R and Schwan H P 1989 Dielectric properties of tissues and biological materials: a critical review Crit. Rev. Biomed. Eng. 17 25-104

Geddes L A and Baker L E 1967 The specific resistance of biological material-a compendium of data for the biomedical engineer and physiologist Med. Biol. Eng. 5 271-93

Gibson A, Tidswell T, Bayford R H and Holder D S 2000 Spherical and realistic head models in electrical impedance tomography Biomedical Applications of EIT 1 Scientific Abstracts 3rd EPSRC Engineering Network Meeting (University College London, 5-7 April 2000, London) pp 150-4

Golias N A and Dutton R W 1997 Delaunay triangulation and 3D adaptive mesh generation Finite Elem. Anal. Des. $25331-41$

Golub G H and O'Leary D P 1989 Some history of the conjugate gradient and Lanczos algorithms SIAM J. Optim. 2(1) 21-42

Hake J F 1992 Parallel algorithms for matrix operations and their performance on multiprocessor systems Advances in Parallel Algorithms ed L Kronsjö and D Shumsherrudin (London: Blackwell) pp 396-437

Holder D S and Brown B H 1993 Biomedical Application of EIT: A Critical Review Clinical and Physiological Applications of EIT ed D S Holder (London: UCL Press)

Jain H, Isaacson D, Edic M P and Newell J C 1997 Electrical impedance tomography of complex conductivity distributions with noncircular boundary IEEE Trans. Biomed. Eng. 44 1051-60

Metherall P, Barber D C, Smallwood R H and Brown B H 1996 Three-dimensional electrical impedance tomography Nature 380 509-12

Molinari M, Blott B H, Cox S J and Daniell G J 2002 Optimal imaging with adaptive mesh refinement in electrical impedance tomography Physiol. Meas. 23 121-8

Molinari M, Cox S J, Blott B H and Daniell G J 2001a Adaptive mesh refinement techniques for electrical impedance tomography Physiol. Meas. 22 91-6

Molinari M, Cox S J, Blott B H and Daniell G J 2001b Efficient non-linear 3D electrical tomography reconstruction Proc. 2nd World Congress on Industrial Process Tomography (29-31 September 2001 Hannover, Germany) 
Shewchuk J R 1994 An Introduction to the Conjugate Gradient Method Without the Agonizing Pain (Pittsburgh, PA: Carnegie Mellon University)

Somersalo E, Cheney M and Isaacson D 1992 Existence and uniqueness for electrode models for electric current computed tomography SIAM J. Appl. Math. $521023-40$

Takeda A L, Cox S J and Payne A J 2000 Parallel numerical modelling of ice flow in Antarctica Arabnia H R, Proc. Int. Conf. Parallel and Distributed Processing Techniques and Applications (June 2000) Las Vegas, NV, CSREA Press pp 335-41

Vauhkonen M, Lionheart W R B, Heikkinen L M, Vauhkonen P J and Kaipio J P 2001 A MATLAB package for the EIDORS project to reconstruct two-dimensional EIT images Physiol. Meas. 22 107-11

Vauhkonen P J, Vauhkonen M, Savolainen T and Kaipio J P 1999 Three-dimensional EIT based on the complete electrode model IEEE Trans. Biomed. Eng. 46 1150-60

Yorkey T J, Webster J G and Tompkins W J 1987 Comparing reconstruction algorithms for electrical impedance tomography IEEE Trans. Biomed. Eng. 34 843-52 